baseline and periodically for at least 6 months postexposure (eg, 6 weeks, 12 weeks, 6 months). If PEP is used, drug toxicity should be monitored.

These recommendations by the interagency group are provisional, because they are based on limited data regarding efficacy and toxicity of PEP and risk of HIV infection after exposure. Because the majority of occupational exposures to HIV do not result in infection transmission, potential toxicity must be considered carefully when prescribing PEP. The recommendations should be implemented in consultation with persons having expertise in antiretroviral therapy and HIV transmission.

The CDC urges enrollment of all workers in the US who receive $\mathrm{PEP}$ in an anonymous registry being developed by the CDC and the Glaxo Wellcome Company to assess toxicity. Unusual or severe toxicity from antiretroviral drugs should be reported to the manufacturer and the FDA. Starting in early 1997, updated information on HIV PEP will be available from the CDC internet home page (www.cdc.gov); fax information service (404-332-4565, Hospital Infections Program directory); National AIDS Clearinghouse (800-458-5231); and HIV/AIDS Treatment Information Service (800-448-0440). (See SHEA News, "Postexposure Antiretroviral Prohylaxis.")

FROM: Centers for Disease Control and Prevention. Update: provisional recommendations for chemoprophylaxis after occupational exposure to HIV. MMWR June 9, 1996;45:468-472.

\section{New HCV Exposure Guidelines}

The CDC has revised its guidelines for follow-up after occupational exposure to hepatitis $\mathrm{C}$ virus (HCV), citing risk of both occupational and nosocomial transmission of HCV.

In summarizing the results of follow-up studies of HCWs who sustained percutaneous exposures to blood from anti-HCV-positive patients, the $\mathrm{CDC}$ noted that the incidence of anti-HCV seroconversion (based on second-generation testing) averaged $3.5 \%$ (range, $0 \%$ to $7 \%$ ); in the one study that used polymerase chain reaction to measure $\mathrm{HCV}$ infection by detecting HCV RNA, the incidence was $10 \%$.

The CDC also noted that hospitalized patients may serve as a reservoir for transmission; the prevalence of anti-HCV among patients has been reported to range from $2 \%$ to $18 \%$. A number of nosocomial outbreaks also were summarized. In one report from Australia, four patients who had outpatient surgery on the same day became infected with HCV of the same genotype as a chronically infected patient who underwent surgery just prior to the cases. In a report from Spain, five open-heart-surgery patients acquired $\mathrm{HCV}$ infection from a cardiovascular surgeon with chronic HCV.

In the absence of postexposure prophylaxis, there are multiple issues that need to be considered in deciding if there should be a defined protocol for the follow-up of HCWs for HCV infection after occupational exposure. These include the limited data on the risk of transmission, the limitations of available serological testing for detecting infection and determining infectivity, the poorly defined risk of transmission by sexual, household, and perinatal exposures, the limited benefit of therapy for chronic disease (eg, alpha interferon), the medical and legal implications, and the cost of follow-up. The CDC has estimated the nationwide cost of providing postexposure follow-up testing at $\$ 2$ to $\$ 4$ million per year; the cost per person for each person who benefits from therapy is estimated at $\$ 200,000$.

In the summary of recommendations, the CDC stated that no postexposure prophylaxis is available for hepatitis $\mathrm{C}$ and that immune globulin is not recommended because it does not appear to be effective in preventing hepatitis $\mathrm{C}$. The CDC recommended that institutions should provide $\mathrm{HCWs}$ with accurate and up-to-date information on the risk and prevention of all bloodborne pathogens, including hepatitis $\mathrm{C}$. In addition, institutions should consider implementing policies and procedures for follow-up of HCWs after percutaneous or mucosal exposure to anti-HCV-positive blood. Such policies might include baseline testing of the source patient for anti-HCV and baseline and 6-month follow-up testing of the persons exposed for anti-HCV and alanine aminotransferase activity. All anti-HCV results should be confirmed by supplemental anti-HCV testing.

The issue of the HCV-infected HCW also is addressed, and the guidelines state that the risk of transmission from an infected worker to a patient appears to be very small and that there currently are no recommendations regarding restriction of HCWs with hepatitis $\mathrm{C}$. As recommended for all $\mathrm{HCWs}$, those who are anti-HCVpositive should follow strict aseptic technique and standard (universal) precautions, including appropriate use of handwashing, protective barriers, and care in the use and disposal of needles and other sharp instruments.

A copy of this document may be obtained from the CDC Hepatitis Surveillance Branch (telephone 404-6393408).

FROM: Centers for Disease Control and Prevention. Issues and answers: what is the risk of acquiring hepatitis $\mathrm{C}$ for health care workers and what are the recommendations for prophylaxis and follow-up after occupational exposure to hepatitis C virus? Centers for Disease Control and Prevention: Hepatitis Surveillance Report: No. 56; April 1996.

\section{Fatal Toxemia of Dialysis Patients}

An outbreak of severe toxic reactions among 131 dialysis patients occurred at a dialysis center in Caruaru, near Recife, in northern Brazil. Between February 17 and 20, 1996, patients reported visual disturbances, abdominal pain, and vomiting associated with dialysis. On February 20 , one patient died soon after completing a dialysis session. Between February 22 and March 6, 1996, 11 additional patients died; the Ministry of Health was notified, and the center was closed. Surviving patients initially were transferred to the city's other dialysis center, but now are being dialyzed outside of Caruaru. As of May 13, 46 patients were known to have died, and over 40 others have been hospitalized in Recife. Over $90 \%$ of the patients at the affected dialysis center reported having visual disturbances, gastrointestinal complaints, and muscle weakness; 